\title{
Customer portfolio management using Z-ranking of customer segments and the LTV perturbation method
}

Received (in revised form): 26th March, 2007

\section{Ram Gopalan}

is a faculty member at the Fox School of Business, Temple University, Philadelphia. His research interests include formulating customer management strategy, calculating ROI on marketing investments and assessing the efficacy of distribution channels.

Keywords customer portfolio management, lifetime value modelling, marketing optimisation, portfolio simulation, segmentation

\begin{abstract}
Customer lifetime value (LTV) models have long been used to focus direct marketing campaigns by allocating resources to those customers who are deemed to offer the most value to the company. Traditionally, customers are rank-ordered by their lifetime value and more marketing resources are targeted towards the customers with the greatest value. In this paper, a framework for visualising customer segments, called Z-ranking, is developed, along with a method for customer portfolio management, called the Life Time Value Perturbation Technique. Rather than viewing a customer's LTV as a quantity that is immutable, this framework adopts the point of view that the LTV can be perturbed, via application of appropriate customer management strategies. Through a series of 'What-if' scenario analyses, a firm can focus scarce resources on those investments that lead to the greatest increase in the value provided by a customer portfolio. The LTV perturbation technique is further embedded in a four-phase procedure that forms the basis for customer portfolio management, enabling the strategic marketer to continually identify and improve customer management strategies that lead to the greatest impact on direct marketing performance.

Journal of Database Marketing \& Customer Strategy Management (2007) 14, 225-235. doi:10.1057/palgrave.dbm.3250050
\end{abstract}

\section{INTRODUCTION}

The lifetime value (LTV) model is a topic that is currently of great interest to both database marketing practitioners and the academic community. Jain and Singh ${ }^{1}$ provide a comprehensive review of research pertaining to assessment of a customer's lifetime value. In the past, the LTV model has been mostly used as a mechanism to rank-order prospects in a database. In lieu of scattering marketing resources by soliciting prospects indiscriminately, database marketers have used the LTV model as a mechanism to sift through the database and identify higher value customers. ${ }^{2}$

Venkatesan and $\mathrm{Kumar}^{3}$ present an interesting use of the LTV model to prioritise marketing effort in a B-to-B context. Berry ${ }^{4}$ has devised a decision engine for campaign planning that takes a sophisticated, longitudinal view of a customer over multiple planning periods, accounting for many important constraints such as contact interval rules and segment 
communication protocols. He reports significant benefits from use of the decision engine, with the additional advantage that the methodology requires no operational software changes. A similar approach to marketing optimisation is reported by Williams. ${ }^{5}$ This optimisation approach also takes a longitudinal view of a customer over multiple planning periods and considers many dimensions of campaign planning such as offers, recipients, timing and channels. Campaign budget goals can be incorporated in both Berry ${ }^{4}$ and Williams ${ }^{5}$ and both methodologies are capable of extensive customisation of the campaign to individual recipients.

Many algorithms for building a profitability model assume that the LTV must be estimated for a single customer. Recently, however, Yang ${ }^{6}$ has proposed a concept called lifetime value average (LTVA) that measures the aggregate value of an entire portfolio of customers. The portfolio approach to customer management has also been examined in other works such as of Ang and Taylor. ${ }^{7}$

In a direct marketing context, decisionmakers face conflicting objectives; multiple projects clamouring for resources and stringent goals for portfolio profitability and customer base growth. In such situations, managers must answer such routinely asked questions as 'is it better to increase cross-sell rates for existing, profitable customers, or is it better to increase the campaign response rate for a potentially lucrative customer segment that has historically consisted of poor responders, or is it better to focus resources on tighter account management for an existing customer segment prone to high-default rates'? The Z-ranking framework and the $L T V$ perturbation method together represent a disciplined approach to rank-ordering such alternatives, based upon a systematic assessment of their overall impact on the entire customer portfolio.

In this paper, there are also two sharp philosophical deviations from previous LTV research. First, instead of viewing a customer's LTV as a static and immutable entity, the lifetime profitability calculation is embedded in a model whose parameters can be perturbed, at least moderately, along some dimensions. The LTV perturbation method forms the basis for selecting customer management strategy, providing the marketer with a systematic mechanism to identify the profitability levers for an entire portfolio of customers. Secondly, the LTV model is viewed as an attribute of a customer segment rather than an individual customer and it is this 'segment-based' LTV model that will be perturbed. The LTV perturbation method is embedded in a four-phase methodology, to examine and adopt the most suitable perturbation opportunities.

\section{THE Z-RANKING FRAMEWORK FOR VISUALISING CUSTOMER SEGMENTS}

Marketers have historically adopted many different types of segmentation, including use of demographic variables such as age and income, psychographic variables including lifestyles, attitudes, interests and opinions, and behavioural variables such as product usage rate. Some desirable properties of customer segments are that they be stable over time and easily identifiable. A framework for representing customer segments and the overall value provided by a customer portfolio is depicted in Figure 1. All customers are classified into one of the four quadrants, representing combinations of their relationship level (prospect vs existing customer) and profitability level (high vs low). Customers affording a profit that is higher than a predetermined threshold $P_{\mathrm{T}}$ are deemed to be 'high-profit' customers. Marketers derive the greatest value from existing, high-profit customers (quadrant 1) and the least value from low-profit prospects (quadrant 4). The progression of decreasing value, from quadrant 1 to quadrant 4 , as depicted in 


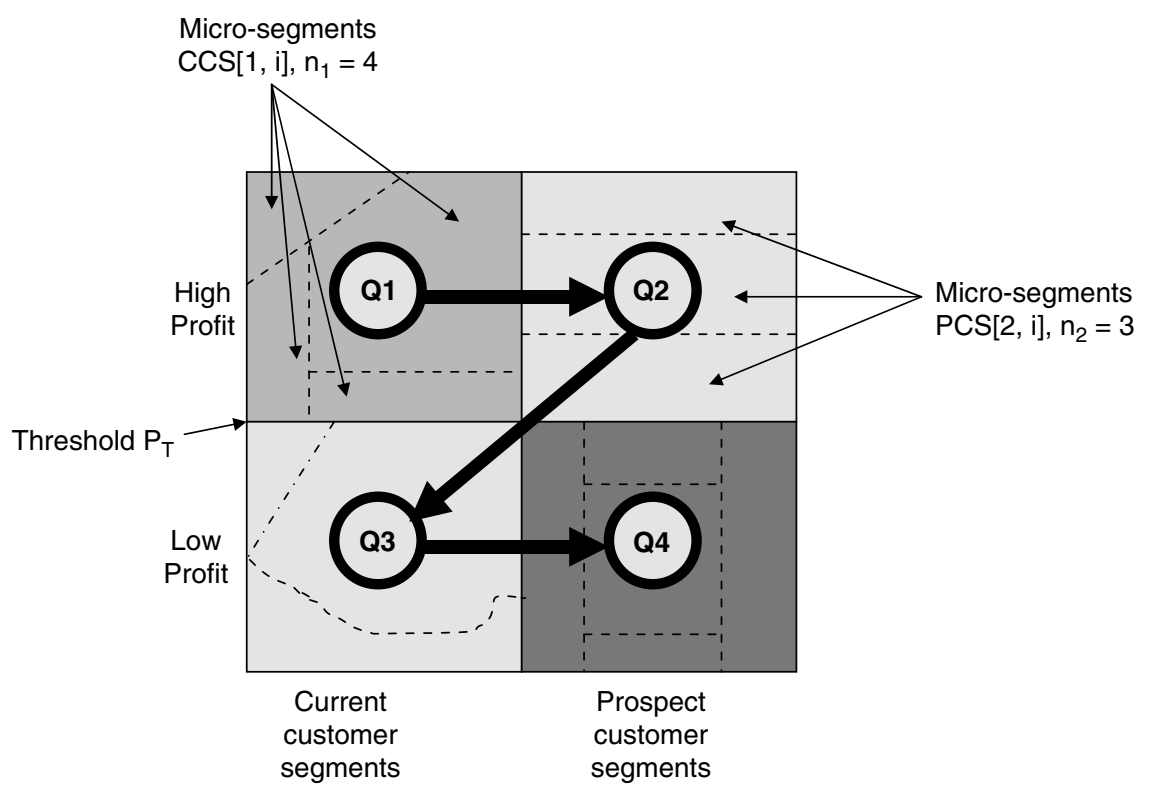

Figure 1: The four quadrants in the Z-ranking framework for customer segments

Figure 1, looks like the letter Zeta, and we refer to the framework as a $Z$-ranking of customer segments. The customers within each quadrant are further subdivided into micro-segments, using segmentation methodologies and variables that could indeed vary from quadrant to quadrant. There are $n_{k}$ micro-segments within quadrant $k$. In Figure 1, $n_{1}=4, n_{2}=3, n_{3}=3$ and $n_{4}=5$. Quadrants 1 and 3 are referred to as current customer segments (CCS) and quadrants 2 and 4 are prospect customer segments (PCS). The following parameters are of interest to the database marketer and tracked periodically, for example, every quarter or between campaigns.

N_PCS $[2, i]$, N_PCS $[4, i]=$ the number of prospects that are to be mailed an offer in a particular micro-segment $i$, in quadrants 2 and 4 , respectively.

VAL_PCS[2, i],VAL_PCS[4, i] = Average value of a customer in prospect microsegment $i$, in quadrants 2 and 4 , respectively.

N_CCS[1,i], N_CCS[3,i] = Number of current customers in micro-segment $i$, in quadrants 1 and 3, respectively.
VAL_CCS $[1, i]$, VAL_CCS $[3, i]=$ Average value of a current customer in microsegment $i$, in quadrants 1 and 3 , respectively.

The total customer portfolio value (TCPV) can then be represented by the following equation:

$$
\begin{aligned}
\text { TCPV }= & \Sigma_{\text {PCS_Q2 }}\{\text { N_PCS }[2, i] \\
& \times \text { VAL_PCS }[2, i]\}+\Sigma_{\text {PCS_Q4 }} \\
& \times\left\{\text { N_PCS }[4, i] \times V A L \_P C S[4, i]\right\} \\
& +\Sigma_{\text {CCS_Q1 }}\left\{\mathrm{N} \_C C S[1, i]\right. \\
& \times \text { VAL_CCS }[1, i]\}+\Sigma_{\text {CCS_Q3 }} \\
& \times\left\{\text { N_CCS }[3, i] \times V A L \_C C S[3, i]\right\}
\end{aligned}
$$

A database marketer's campaign goals can be paraphrased using the Z-ranking framework as follows: to reach a target customer portfolio value TCPV, while simultaneously attaining micro-segment profitability goals, stated in terms of target values for $\mathrm{N}_{-}$ PCS $[2, i]$, N_PCS[4, i],VAL_PCS_[2, $i]$, VAL_PCS[4, $i]$, N_CCS[1, $i]$, N_CCS[3, $i]$, VAL_CCS $[1, i]$ and VAL_CCS[3, $i]$, for each micro-segment $i$, in any quadrant. To increase TCPV, the marketer can increase the number of customers in a micro-segment 
or work on enhancing the value afforded by each customer in a segment. It is assumed that the value of a customer can be inferred from a suitable LTV model that is representative of the 'median' customer in the relevant micro-segment. In Figure 1, $\left(n_{1}+n_{2}+n_{3}+n_{4}\right)=15$ LTV models must be built in total, to represent the entire universe of customers. Moreover, the parameters that drive the value of the LTV model can be perturbed (within limits), to alter the value provided by the 'average' customer in the micro-segment. Thus, the goal of the database marketing campaign is not only to increase the number of customers in a micro-segment, but also to suitably perturb the value provided by each customer in the micro-segment. The LTV perturbation method described in the next sections completely outlines the methodology.

\section{THE FOUR PHASES OF THE LTV PERTURBATION METHOD}

I shall illustrate the method using an example drawn from the credit card industry. The example will also serve to make the point that exercising the LTV model in any form requires considerable customisation to the business context at hand. Consider a company selling two charge-card products, a lower-profit product (I) and a higher-profit product (II). In any database marketing campaign, a prospect is typically offered Product I first and if she proves profitable enough, the customer may be targeted for cross-selling Product II later on. For both products, the customer is not permitted to revolve balances (this is done merely for ease of exposition). The drivers of profitability for the two charge-card products are:

- A discount revenue provided by merchants, that is based upon the customer's annual charges. The discount revenue is stated as a per cent (henceforth DR\%) of the annual charge volume.

- An annual fee, stated in dollars.
- Any losses accrued in the event that the customer defaults on payment. The default amount is typically assumed to be a fraction of the annual spend, for example, if the company loses two months of customer spend in the event of default, the default amount is calculated as (Annual Spend/12) $\times 2$.

Table 1 summarises the profitability assumptions for products I and II.

\section{Phase I: Identify micro-segments in each quadrant of the Z-ranking framework}

The first phase provides a mechanism for the decision-maker to envision the entire customer portfolio as a set of mutually exclusive, collectively exhaustive customer micro-segments. The first task is to determine a threshold profit level $P_{\mathrm{T}}$ (see Figure 1), and then to classify customer segments into the four quadrants (if need be, the entire four phase process can be repeated with varying levels of $P_{\mathrm{T}}$ to examine sensitivity of the procedure to this threshold). Once $P_{\mathrm{T}}$ has been determined, each quadrant is envisioned as a collection of customer micro-segments, consisting of relevant $\mathrm{PCS}[k, i]$ or CCS[ $k, i]$, where the ordered pair $[k, i]$ denotes the $i$ th micro-segment in quadrant $k$. The exact segmentation methodology used to form micro-segments PCS $[k, i]$ and CCS $[k, i]$ is not central to the LTV perturbation method per se. The only requirement is that each customer micro-segment identified by the methodology be amenable to the construction of a robust LTV model that is representative of the 'average' customer who belongs to the micro-segment. Some common segmentation approaches are listed below.

Table 1: Product feature profile for I and II

\begin{tabular}{lll}
\hline Product \# & $\begin{array}{l}\text { DR\% paid by } \\
\text { merchants }\end{array}$ & $\begin{array}{l}\text { Annual fee paid } \\
\text { by cardholders }\end{array}$ \\
\hline Product I & 1.5 & $\$ 25$ \\
Product II & 2 & $\$ 80$ \\
\hline
\end{tabular}




\section{RFM segmentation}

The RFM methodology classifies customers based upon the recency, frequency and monetary value of their purchases. The papers of Shih and $\mathrm{Liu}^{2}$ and Venkatesan and Kumar ${ }^{3}$ are exemplars of using an RFM analysis. While the RFM methodology has the advantage of simplicity, it is best used for classifying existing customers rather than prospects.

\section{Behavioural/tenure/risk profile-based segmentation}

A slight generalisation of RFM segmentation, behavioural segmentation can classify customers based upon their spending patterns, tenure with the company or their risk profile. Banks and credit card companies in particular, price their products to certain customers based upon their risk profile, as expressed in their FICO score (for more information, refer to http://www.myfico.com). It is also common for credit providers to segment their portfolios based upon client tenure, for example, more stringent account management principles may be adopted for customers with lower tenure.

\section{Cluster analysis}

This methodology is an example of a 'noncriterion'-based segmentation technique. ${ }^{8}$ The customers within any single cluster are very similar to each other (and customers in different clusters are very dissimilar to each other). The segments identified by cluster analysis are derived from demographic descriptors such as age and income.

Customers in different clusters are not guaranteed to be vastly different with respect to some important criterion variable such as profitability. Nevertheless, cluster analysis remains important to the marketer and has the advantage of simplicity of execution.

\section{CHAID segmentation}

The CHAID segmentation technique is discussed in detail by Magidson. ${ }^{8}$ CHAID is a 'criterion'-based segmentation technique, and customers in different
CHAID clusters are guaranteed to differ with respect to some benchmark metric such as profitability. If the goal of LTV modelling is to identify a unique framework that represents the profitability of each customer segment, it may be advantageous to use CHAID as the underlying segmentation methodology.

The following five distinct customer micro-segments are relevant for the credit card example.

Current Customer Segment 1 in Quadrant 1 (CCS [1, 1]): Existing customers for Product I characterised by low risk, high degree of (voluntary) attrition, low propensity to accept cross-sell offers and high spend.

Current Customer Segment 2 in Quadrant 1 (CCS [1, 2]): Existing customers for Product II characterised by very low risk, some degree of (voluntary) attrition and very high spend.

Prospect Customer Segment 1 in Quadrant 2 (PCS $[2,1])$ : Attractive customer prospects for Product I characterised by low risk, high spend and a low propensity to respond to an offer for Product I.

Current Customer Segment 1 in Quadrant 3 (CCS [3, 1]): Existing customers for Product I characterised by high risk, low degree of (voluntary) attrition and high spend.

Prospect Customer Segment 1 in Quadrant 4 (PCS [4, 1]): Customer prospects for Product I characterised by high risk, high spend and high propensity to respond to an offer for Product I.

The relative sizes of CCS[1, 1], CCS[1, 2] and CCS[3,1] are known precisely and are denoted by N_CCS[1, 1], N_CCS[1, 2] and N_CCS[3, 1]. It is to be noted that these segment sizes are slightly mutable quantities, especially since customers can move from one segment to another, for example, from CCS $[1,1]$ to CCS $[1,2]$, via a cross-sell offer. Moreover, it is assumed that the database marketer has determined target values for the number of new prospects to be mailed in prospect segments PCS[2,1] and PCS[4, 1]; these quantities, N_PCS[2, 1] and N_PCS[4, 1], can also be inferred from 
prior campaigns, if a suitable database of prior campaigns is available.

\section{Phase II: Formulate a robust LTV model for each micro-segment}

The goal of Phase II is to formulate a robust LTV model that is representative of each customer micro-segment identified in Phase I. Ideally, each segment's model should possess the following property:

(LTV inferred from the model) $\times(\#$ customers in micro-segment)

$=$ Total value of customer micro-segment

The notion here is that the LTV model for a segment represents the value of the 'average' customer in the segment, similar to the concept of the LTVA. ${ }^{6}$ The important stylistic difference in this paper is that each segment's LTV model can be studied with associated model parameters, for example, campaign response rate and spend per year, and it is these model parameters that will be 'perturbed' in Phase III of the method.
To continue with the credit card example, a generic LTV model template, that can be adapted for all customer microsegments identified in Phase I, is stated in Figure 2. At time $t=0$, a prospect is sent an offer for Product I. If she accepts the offer (with probability $p_{1}$ ), she may make one of the following four decisions exactly at the end of the first year of her tenure: (i) accept a cross-sell for product II (with probability $p_{2}$ ); (ii) make no change and retain product I for a second year $\left(p_{3}\right)$; (iii) cancel the credit card after paying down all balances $\left(p_{4}\right)$ or (iv) default on payment, taking a few months of spend $\left(p_{5}\right)$. If she stays for a second year, the decision tree describes a further sequence of events, based upon the product that she holds for the second year. The LTV calculations are truncated after a two-year time horizon for this illustrative example, even though it is a common practice to evolve the LTV decision tree for a five-year period. As a consequence, in state $S_{1}$, the value derived from the customer during year 3 is not included in

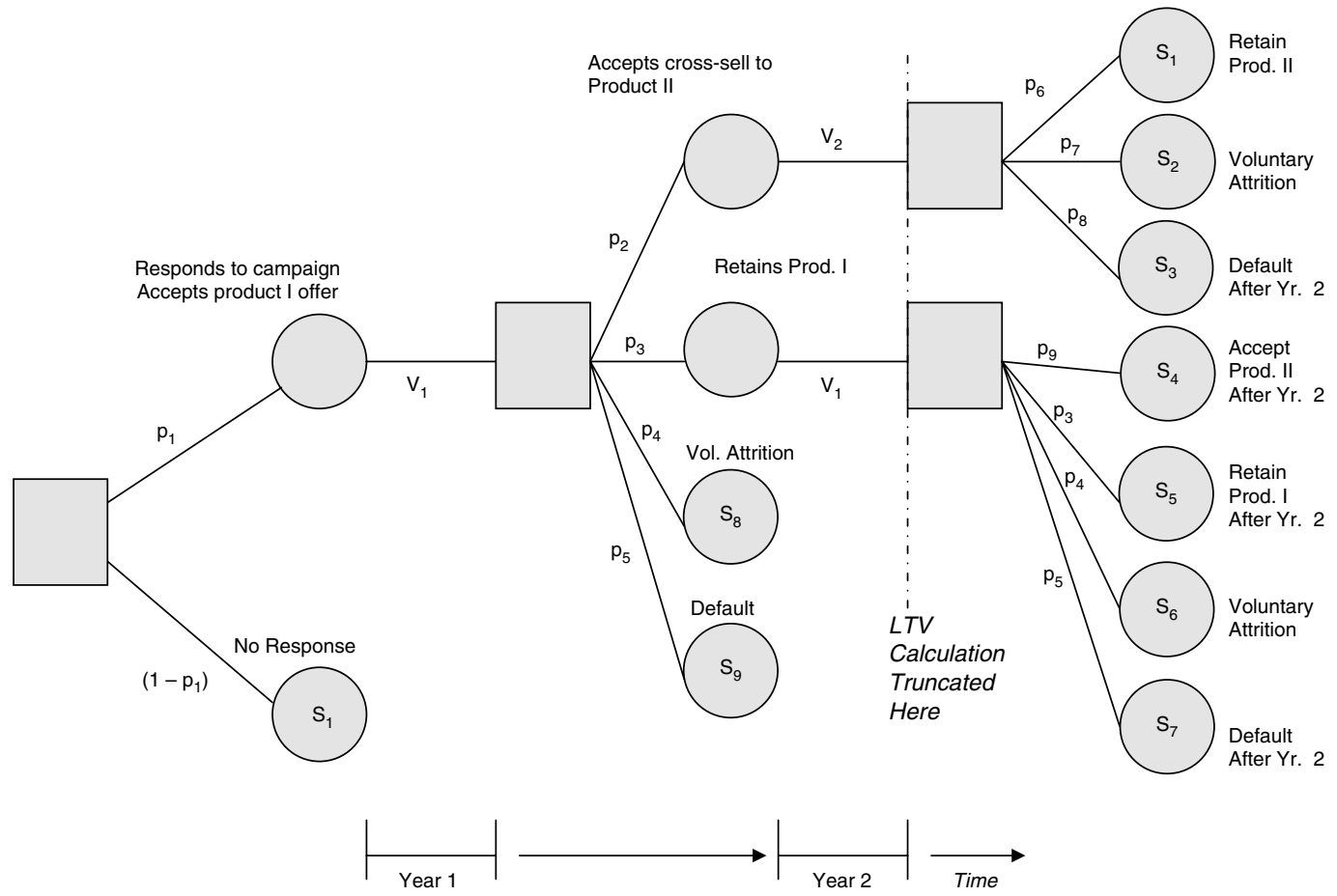

Figure 2: A LTV decision tree spanning a two-year period 
the calculation, but in state $S_{3}$ the loss due to default, incurred exactly at the end of a two-year epoch, is included in the LTV calculation. Table 2 provides a summary of every possible state, with associated probability and customer value. The final LTV is determined as:

$$
\text { LTV }=\Sigma_{S i}\left\{\text { Probability }\left(S_{i}\right) \times \text { Value }\left(S_{i}\right)\right\}
$$

The LTV template from Figure 2 can be adapted to describe all five customer microsegments identified in Phase I. Table 3 provides a description of the behavioural characteristics of the five segments and by combining the

Table 2: Summary of all possible scenarios in a two-year life time value tree

\begin{tabular}{lll}
\hline Scenario \# & $\begin{array}{l}\text { Probability of } \\
\text { scenario }\end{array}$ & $\begin{array}{l}\text { Customer value } \\
\text { under scenario }\end{array}$ \\
\hline$S_{1}$ & $p_{1} p_{2} p_{6}$ & $V_{1}+\left\{V_{2} /(1+r)\right\}$ \\
$S_{2}$ & $p_{1} p_{2} p_{7}$ & $V_{1}+\left\{V_{2} /(1+r)\right\}$ \\
$S_{3}$ & $p_{1} p_{2} p_{8}$ & $V_{1}+\left\{V_{2} /(1+r)\right\}$ \\
& & $-\{($ Default_Amount \\
& $\left.\left.\left.p_{3}\right)\right) /(1+r)\right\}^{*}$ \\
$S_{4}$ & $p_{1} p_{3} p_{2}$ & $V_{1}+\left\{V_{1} /(1+r)\right\}$ \\
$S_{5}$ & $p_{1} p_{3} p_{4}$ & $V_{1}+\left\{V_{1} /(1+r)\right\}$ \\
$S_{6}$ & $p_{1} p_{3} p_{5}$ & $V_{1}+\left\{V_{1} /(1+r)\right\}$ \\
$\left.S_{7} /(1+r)\right\}$ \\
& & $-\{($ DefaultAAmount \\
& $p_{1} p_{4}$ & $\left.\left.\left(S_{7}\right)\right) /(1+r)\right\}^{\top}$ \\
$S_{8}$ & $p_{1} p_{5}$ & $V_{1}$ \\
$S_{9}$ & $\left(1-p_{1}\right)$ & Amount(S) $\}^{\top}$ \\
$S_{10}$ & $($ Default \\
\hline
\end{tabular}

*Default_Amount $\left(S_{3}\right)=(2 / 12) \times$ Annual Customer Segment Spend for Product II (refer Table 3).

${ }^{\dagger}$ Default_Amount $\left(S_{7}\right)=$ Default_Amount $\left(S_{9}\right)=(3 / 12)$ $\times$ Annual Customer Segment Spend for Product I (refer Table 3).

The acquisition (solicitation) cost for all customers is assumed to be $\$ 1$. information from Tables 1-3, a precise customer LTV can be stated for a generic customer in each micro-segment, as stated below.

$$
\begin{aligned}
& \text { VAL_PCS }[2,1]=\$ 25.54, V A L \_P C S[4,1] \\
& =\$ 17.12, \text { VAL_CCS }[1,1] \\
& =\$ 275.52 \text {,VAL_CCS }[1,2] \\
& =\$ 704.71, \text { VAL_CCS[3,1] }=\$ 17.57
\end{aligned}
$$

It is to be noted that the LTV model treats customers in micro-segment CCS[1, 1] similar to prospects in micro-segment PCS[2, 1], but with $p_{1}$ set equal to 1 in Table 3 . Hence, the value of customers in micro-segment CCS[1,1] is much higher than the value of customers in micro-segment PCS[2,1]. Such a valuation is consistent with the findings of previous research, for example, Reichheld, ${ }^{9}$ which has pointed out that retaining existing customers is more important to the long-term profitability of a firm, when compared to recruiting new prospects. LTV modelling therefore provides a neat mechanism to quantify the superior value that an existing customer offers to a firm (compared to a prospect).

\section{Phase III: Identify LTV model perturbation opportunities and their impact}

The next step is to identify various options for increasing TCPV. Each of these options in essence attempt to increase either $\mathrm{N}$ or VAL for a micro-segment, using a LTV model perturbation. Each perturbation

\begin{tabular}{|c|c|c|c|c|c|c|c|c|c|c|c|c|c|}
\hline Segment $^{\star}$ & $\begin{array}{l}\text { Product } \\
\text { held }\end{array}$ & $\begin{array}{l}\text { Annual } \\
\text { spend }\end{array}$ & $\begin{array}{l}\text { Fraction of product's } \\
\text { annual spend lost } \\
\text { during default }\end{array}$ & $V_{1}$ & $V_{2}$ & $P_{1}$ & $P_{2}$ & $P_{3}$ & $P_{4}$ & $P_{5}$ & $P_{6}$ & $P_{7}$ & $P_{8}$ \\
\hline $\operatorname{PCS}(2,1)$ & I & $\$ 5000^{\dagger}$ & $(3 / 12)$ & $\$ 100$ & NA & 0.15 & 0.04 & 0.90 & 0.05 & 0.01 & 0.9 & 0.1 & 0.0 \\
\hline $\operatorname{PCS}(4,1)$ & I & $\$ 5000$ & $(3 / 12)$ & $\$ 100$ & NA & 0.30 & 0.00 & 0.90 & 0.05 & 0.05 & NA & NA & NA \\
\hline $\operatorname{ccs}(1,1)$ & I & $\$ 8000^{\dagger}$ & (3/12) & $\$ 145$ & NA & 1.0 & 0.02 & 0.90 & 0.08 & 0.00 & 0.9 & 0.1 & 0.0 \\
\hline $\operatorname{CcS}(3,1)$ & I & $\$ 6000$ & $(3 / 12)$ & $\$ 115$ & NA & 1.0 & 0.00 & 0.90 & 0.03 & 0.07 & NA & NA & NA \\
\hline $\operatorname{ccs}(1,2)$ & II & $\$ 15,000$ & $(2 / 12)$ & NA & $\$ 380$ & NA & NA & NA & NA & NA & 0.9 & 0.1 & 0.0 \\
\hline
\end{tabular}
opportunity is denoted as PERT $j]$, for $j=1, \ldots, m$. In the discussion considered below, $m=7$.

Table 3: Spend/risk profile for the customer segments

*Segments in quadrants 1 and 2 are high profit, that is, $\operatorname{PCS}(2,1), \operatorname{CCS}(1,1)$ and $\operatorname{CCS}(1,2)$.

${ }^{\dagger}$ For segments $\operatorname{PCS}(2,1)$ and $\operatorname{CCS}(1,1)$, it is assumed that customers will spend $\$ 15,000 /$ year in year 2 (same as annual spend of CCS(1, 2)), if they accept a cross-sell to Product II at the end of year 1. 
PERT[1]: Increase spend/year for segment CCS[1, 1] by 10 per cent, from $\$ 8,000$ to $\$ 8,800$ per year. The marketer can increase share of wallet (spend) for a customer via many mechanisms, for example, sweepstakes. Such a perturbation entails fixed investments in designing new business processes or IT systems and the budget required for this initiative is denoted as $B$ [1]. The impact on the LTV for the segment is that it moves from $\$ 275.52$ per customer to $\$ 297.81$ per customer, an increase of $\$ 22.29$ per customer in CCS $[1,1]$. The weighted impact on TCPV can be obtained by multiplying $\$ 22.29$ by N_CCS $[1,1]$. Assuming that N_CCS $[1,1]=5,000,000$, the overall impact of PERT[1] is $\$ 111,450,000$, in incremental portfolio value. The total incremental portfolio value created by PERT $[j]$ is denoted as $C[j]$, that is, $\mathrm{C}[1]=\$ 111,450,000$. It is also assumed that the budget for executing PERT[1], that is, $\mathrm{B}[1]=\$ 10,000,000$.

PERT [2]: The marketer can attempt to increase the response rate for prospects in PCS[2, 1], via investment in better quality lists or simply by working hard on designing a better offer or even by making up better creativity for the same offer. It will be assumed that response rates for PCS $[2,1]$ can be perturbed by 10 percentage points, from 15 to 25 per cent. The LTV goes up to $\$ 43.24$ from $\$ 25.54$, an increase of $\$ 17.7$ per prospect contacted. The increase in TCPV (assuming the number of mailings is retained constant), is $\$ 17.7$ per customer mailed. The impact on TCPV is $\$ 17.7 \times$ N_PCS[2,1] $=\$ 35,400,000$, assuming N_PCS[2,1] $=2,000,000$ mailings. The budget $B[2]$ for this perturbation is $\$ 2,000,000$ as it entails a purchase of very high-quality lists.

PERT [3]: Reduce the voluntary attrition rates for CCS[1, 1], for example, by investing in better customer complaint management systems. This perturbation reduces the voluntary attrition rate $p_{4}$ from 8 to 4 per cent, while increasing the product retention rate $p_{3}$ from 90 to 94 per cent (or alternately $p_{3}$ could increase to 92 per cent, while $p_{2}$, the cross-sell rate, improves to 4 per cent, but this option is not considered). The impact per customer is to increase the LTV from $\$ 275.52$ to $\$ 281.05$, an increase of $\$ 6.47$ per customer. While the increase in LTV for this perturbation is modest, CCS[1,1] is likely to be the largest micro-segment of the current customer portfolio. Assuming that N_CCS $[1,1]=5,000,000$, the portfolio impact of the perturbation will be $\$ 32$, 350,000 . The cost $B[3]$ of the perturbation is $\$ 2,000,000$.

PERT [4]: Reduce the default rate for $\operatorname{CCS}[3,1]$ from 7 to 2 per cent and the customer retention rate improves to 95 per cent. Such an initiative requires tighter account management procedures and increased collection efforts. The LTV of CCS[3, 1] increases to $\$ 160.91$ from $\$ 17.57$, an increase of $\$ 143.34$ per customer. If N_CCS[3,1] $=300,000$, then the portfolio impact will be $\$ 43,002,000$. The cost $B[4]$ of the perturbation will be $\$ 1,000,000$.

PERT [5]: Increase the cross-sell rate for customers in CCS[1, 1]. While this perturbation has an immediate impact on CCS[1, 1], it may also improve the LTV of models for PCS[2, 1], PCS[4, 1] and $\operatorname{CCS}[3,1]$ in the long run. It is assumed that $p_{2}$ improves from 2 to 6 per cent for customers in $\operatorname{CCS}[1,1]$ because of this perturbation. As a result, $p_{3}$ reduces to 87 per cent and $p_{4}$ reduces to 7 per cent (in this sense, PERT[5] is similar to PERT[3]). The increase in LTV is $\$(285.86$ $\$ 275.52)=\$ 10.34$ and since N_CCS $[1,1]$ is $5,000,000$, the portfolio impact will be $\$ 51,700,000$. It is assumed that $B[5]=\$ 3,000,000$.

PERT[6]: Increase the incentives for referrals and viral marketing, thereby lowering customer acquisition cost. Such an initiative does not change the structure of any of the LTV models (and their values), 
but does increase N_CCS[1, 1]. It is assumed that 5 per cent of customers in CCS[1,1] refer new customers who are acquired at no cost and become part of micro-segment CCS[1,1] (the exact proportion can be verified through a small test campaign which will be accounted for in the cost $B[6])$. Since N_CCS $[1,1]=5,000,000$, this results in 250,000 new customers at a value of $\$ 275.52$ each and the portfolio impact is $\$ 68,880,000$. The cost $B[6]$ is $\$ 8,000,000$.

PERT [7]: Increase the discount revenue (DR\%) for Product I, from 1.5 to 2.0 per cent. While such a perturbation increases the overall value afforded by a single customer, it may have other negative consequences, as merchants may discourage use of the product, leading to an overall decline in portfolio spend (unless additional value is offered to the merchant in return for the DR\% increase). A complementary perturbation of a similar nature is a fee increase for Product I, from $\$ 25$ to $\$ 30$. The fee increase will surely have other negative ramifications, for example, a lowered campaign response rate for PCS[2, 1] and $\operatorname{PCS}[4,1]$, as well as a higher voluntary attrition rate, that is, a higher $p_{4}$. Such perturbations, with a wide-ranging portfolio impact, will be referred to as Global Perturbations (ie their impact is not localised to a single customer or a single customer micro-segment). The impact of global perturbations cannot be gauged quantitatively from a LTV model, but must be assessed qualitatively by the marketer. For the ease of exposition, it is assumed here that the credit card company offers merchants some additional value in exchange for the higher discount rate, leading to no diminishment in charge volume. The impact is to raise the LTV of CCS $[1,1]$ to $\$ 349.81$ from $\$ 275.52$ and the portfolio impact is $\$ 371,450,000$. The cost of the perturbation is $\$ 90,000,000$.

Table 4 summarises the seven perturbations, their costs and benefits.

\section{Phase IV: Simulate multiple scenarios for customer portfolio value and select a suitable subset of LTV perturbations}

In Phase IV, the marketer must decide which subset of perturbation opportunities to pursue, given a limited budget. Allocating resources to competing marketing projects is often a contentious and political process, with various departments competing for scarce resources. It is exactly for this reason that $Z$-ranking and LTV perturbations are a valuable exercise, as they attempt to quantify costs and benefits in an objective manner. Some common decision-making approaches for perturbation selection are listed below.

1 Select projects based upon their total portfolio impact (column $C[j]$ in Table 4), till the budget runs out. If this approach is followed, the marketer would first negotiate with merchants to increase the

Table 4: Summary of the perturbations considered, along with cost-benefit

\begin{tabular}{lllll}
\hline Perturbation \# & Description & $\begin{array}{l}\text { Cost } \\
\mathbf{B}[\boldsymbol{j}]\end{array}$ & $\begin{array}{l}\text { Portfolio } \\
\text { impact } \mathbf{C}[\boldsymbol{j}]\end{array}$ & $\begin{array}{l}\text { Portfolio impact/ } \\
\text { dollar spent }\end{array}$ \\
\hline 1 & Increase spend/year for CCS[1,1] & $\$ 10,000,000$ & $\$ 111,450,000$ & $\$ 11.145$ \\
2 & Increase response rates for PCS[2,1] & $\$ 2,000,000$ & $\$ 35,400,000$ & $\$ 17.7$ \\
3 & Reduce voluntary attrition for CCS[1,1] & $\$ 2,000,000$ & $\$ 32,350,000$ & $\$ 16.175$ \\
4 & Reduce default rates for CCS[3,1] & $\$ 1,000,000$ & $\$ 43,002,000$ & $\$ 43.002$ \\
5 & Increase cross-sell rates for CCS[1,1] & $\$ 3,000,000$ & $\$ 51,700,000$ & $\$ 17.23$ \\
6 & Acquire customers via viral marketing & $\$ 8,000,000$ & $\$ 68,880,000$ & $\$ 8.61$ \\
7 & from CCS[1,1] & $\$ 90,000,000$ & $\$ 371,450,000$ & $\$ 4.13$ \\
& Increase discount revenue from & & & \\
\hline
\end{tabular}


discount revenue for product I from 1.5 to 2.0 per cent, then attempt to increase spend/year for customers in segment CSS $[1,1]$ by 10 per cent (from $\$ 8,000$ to $\$ 8,800$ ) and so on.

2 Rank-order and select projects based upon the benefit provided per dollar spent, till the total marketing budget $\mathbf{B}$ is exhausted. The key metric to be evaluated is the ratio in the last column of Table 4. With this approach, the marketer would first attempt to reduce the default rates for $\operatorname{CCS}[3,1]$ and then attempt to increase the campaign response rates for segment PCS[2,1]. It is sometimes possible that performing one perturbation can affect the portfolio revenue impact for another perturbation (global perturbations). If all $m$ perturbations under consideration do not have any pair-wise impact on each other, then the perturbation selection problem can be modelled as a knapsack problem, ${ }^{10}$ a well-studied problem in the operational research literature. The marketer's problem can then be stated as the following constrained optimisation problem.

$\operatorname{Max} \Sigma_{j=1, \ldots, m}\{C[j] \times X[j]\}$

Subject to: $\Sigma_{j=1, \ldots, m}\{B[j] \times X[j]\} \leqslant \mathbf{B}$

$X[j]$ is a 0 or 1 integer variable indicating if perturbation $j$ is selected, $B[j]$ is the budget required for executing perturbation $j, C[j]$ is the portfolio benefit of selecting perturbation $j$ and $\mathbf{B}$ is the total marketing budget available.

3 Select perturbations after performing a comprehensive portfolio simulation, using multiple scenarios. In this approach, the impact of a perturbation on the relevant parameter of a LTV model is stated in a distributional form, for example, PERT[2] improves campaign response rates, from 15 per cent to an improved rate that is anywhere between 20 and 25 per cent, with 20 per cent being a pessimistic estimate, 22 per cent being the most likely estimate and 25 per cent being an optimistic estimate (this framework is similar to assumptions made in a project management network). Multiple scenarios can be simulated and in each simulation run, a local search heuristic (eg genetic algorithm or tabu search ${ }^{11}$ ) selects a subset of perturbations for consideration, along with an estimate of the scenario outcome for each perturbation (optimistic, pessimistic or most likely). The impact on LTV and portfolio value (Table 4) is evaluated for each combination of the perturbation set and scenario outcome. The simulation outcomes can also be combined with an interactive multicriteria decision-making procedure, ${ }^{12}$ where decision-maker input is sought to understand trade-offs between conflicting criteria, for example, market share goals and portfolio profitability goals. Group consensus methods, for example, the Delphi method, can also be applied in this phase.

\section{SUMMARY}

This paper introduces two new tools for the database marketer. The Z-Ranking Framework is a tool for visualising customer microsegments, classifying any customer microsegment into one of four quadrants, based upon the segment's profitability (high vs low) and the segment's relationship status (existing customer vs prospects). The second tool is the LTV perturbation method. To apply the perturbation technique, a robust LTV model is first constructed to represent every micro-segment visualised in the Z-Ranking framework. The Total Customer Portfolio Value, TCPV, is described in terms of (i) the LTV models for the micro-segments and (ii) the number of customers already present (or prospected) in every microsegment. Every segment's LTV model is then systematically perturbed, to generate marketing strategies for increasing the value of any single customer in the 
micro-segment (and hence TCPV). The benefit and cost to execute every perturbation is also documented. In the final phase of the LTV perturbation method, the marketer judiciously implements a subset of LTV perturbations, based upon a consideration of the perturbation's efficacy and the available budget.

The LTV perturbation method requires patience, focus and discipline in terms of execution. It is not for every firm. Some pre-requisites for applying the method are that the firm operate in a data-rich environment, with considerable documentation of the history of prior campaigns. The robustness of the LTV models constructed for every microsegment needs to be carefully validated periodically. The LTV perturbation method offers a precise analytic methodology to plan the evolution of a customer portfolio. It can also serve as a tool to achieve consensus in strategic planning exercises.

\section{References}

1 Jain, D. and Singh, S. S. (2002) 'Customer lifetime value research in marketing: A review and future directions', Journal of Interactive Marketing, Vol. 16, No. 2, pp. 34-45.

2 Shih, Y. and Liu, C. (2003) 'A method for customer life time value ranking - Combining the analytic hierarchy process and clustering analysis', Journal of
Database Marketing and Customer Strategy Management, Vol. 11, No. 2, pp. 159-172.

3 Venkatesan, R. and Kumar, V. (2004) 'A customer life time value framework for customer selection and resource allocation strategy', Journal of Marketing, Vol. 68, pp. 106-125.

4 Berry, J (2006) 'The benefits of using a decision engine to optimize campaign planning for direct marketing', Journal of Database Marketing \& Customer Strategy Management, Vol. 13, No. 4, pp. 319-323.

5 Williams, J. (2006) 'Marketing optimization Realizing maximum value from data driven marketing', White Paper, Experian-Scorex, www.experian-scorex.com.

6 Yang, A. (2005) 'Using life time value to gain long term profitability', Journal of Database Marketing and Customer Strategy Management, Vol. 12, No. 2, pp. 142-152.

7 Ang, L. and Taylor, B. (2005) 'Managing customer profitability using portfolio matrices', Journal of Database Marketing and Customer Strategy Management, Vol. 12, No. 4, pp. 298-304.

8 Magidson, J. (1993) 'The CHAID approach to segmentation modeling: CHI-squared automatic interaction detection' in: Bagozzi, R.P. (ed.) 'Advanced methods of marketing research', Blackwell Business Publishing, Oxford.

9 Reichheld, F. (2003) 'The one number you need to grow', Harvard Business Review, Vol. 81, No. 12, pp. $46-54$.

10 Martello, S. and Toth, P. (1990) 'Knapsack problems: Algorithms and computer implementations', John Wiley \& Sons Inc., New York, USA.

11 Glover, F. and Laguna, M. (2004) 'Tabu search', Kluwer Academic Publishers: Boston, MA, p. 408.

12 Koksalan, M., Zionts, S. and Kyksalan, M. (2001) 'Multiple criteria decision making in the new millennium (Lecture Notes in Economics and Mathematical Systems)', Springer-Verlag, Vol. 507. 